\title{
Dilution of precision for mobile location in Non-Line- of-Sight Environments
}

\author{
J. Y. Huang, P. Wang, Q. Wan, L. P. Chiang, and F. H. Choo \\ School of Electrical and Electronic Engineering \\ Nanyang Technological University \\ Singapore \\ jyhuang@ntu.edu.sg
}

\begin{abstract}
We present an analysis of the time of arrival (TOA) and time difference of arrival (TDOA) based positioning methods in Non-Line-of-Sight (NLOS) environments. Single path propagation is assumed. The positioning accuracy is investigated in terms of Dilution of precisions (DOPs) depending on different NLOS cases. We then show that the weighted DOP (WDOP) is equal to the Cramer-Rao Lower Bound (CRLB) when the number of Line-of-Sight (LOS) base stations (BSs) is sufficient (three or more). Some characteristics of the DOP in the Global positioning system (GPS) are introduced to investigate the performance of positioning accuracy. In addition, some new characteristics of the DOP and the relationship of the DOP between the TOA and TDOA based methods are derived. According to these characteristics, the system can improve positioning accuracy through proper choice of optimum BSs and location techniques.
\end{abstract}

\section{INTRODUCTION}

Glocation estimates for Enhanced-911 (E-911) services [1]. This has boosted the research in the field of wireless location as an important public safety feature, which can also add many other potential applications [2]: location-sensitive billing, fraud protection, person/asset tracking, fleet management, intelligent transportation systems (ITS), mobile yellow pages, and wireless system design and for efficient radio resource management, etc.

Conventional geolocation techniques include the time-ofarrival (TOA), timei-difference-of-arrival (TDOA), signal strength (SS), angle-of-arrival (AOA) based methods, or combination of these. Among these localization techniques, TOA and TDOA based methods have attracted much attention [3]. TOA is the one-way signal propagation time from the MS to a BS. Assuming line-of-light (LOS) propagation and zero TOA measurement error, the TOA of each BS provides a circle centered at the BS on which the MS must lie. With three or more BSs, the MS location can be uniquely determined by finding the intersecting point of the TOA derived circles. In TDOA methods, each TDOA measurement defines a hyperbola as possible locations for the MS and the location of the MS is determined from the intersection of two or more TDOA hyperbolae.
The main problem of cellular wireless location systems is the NLOS situation, when the signal arrives at a BS from reflections. There is no direct, or LOS, path. This often happens in an urban environment. Several methods for mitigating NLOS errors have been addressed in the literature [4-11]. The methods for NLOS mitigation considered in [4-6], which requires a time series of range measurements from a moving user, work well when MS is moving. The methods in $[7,8]$ are similar to method field processing for localization. It firstly measures the propagation characteristics of the channel, and then determines the MS location from a database. The method in [9] uses a constrained condition to restrain NLOS errors. The algorithm presented in [10] replaces the nonlinear terms in the measurement equation with a single variable, and adds a loose variable to the equation, in order to simplify the problem and make it a mathematical programming problem. The methods in [11] generally assume that there is a large number of BSs available for location, with only a small subset of those being NLOS.

Despite these prior studies, two basic questions of NLOS geolocation remain unanswered: what is the performance of location algorithms in NLOS environments and how to improve it? The Dilution of precision (DOP) and Cramer-Rao Bound (CRLB) are two types of measures for the positioning accuracy, and are well discussed for the LOS case [12-17]. However, relatively few studies have been reported on the performance analysis for the NLOS case. Recently, the authors in [18] drive the CRLB on geolocation accuracy in mixed NLOS/LOS environments, under the assumption that there are three or more LOS BSs and NLOS errors can be estimated by LOS BSs. The assumption of NLOS errors that can be estimated makes the problem becoming the classical CRLB problem with Gaussian noise. However, this is a special case in practice environments. More general case is that measurements from all BSs are corrupted by NLOS errors $[6,8,22]$. In this paper, we analyze the performance of the TOA and TDOA based on DOPs in NLOS environments, which only need the mean and variance of range errors. We assume single-path propagation in practice environments. This means that in the presence of multipath signals we need to 
select the strongest or first arriving component. Contributions of our study include the following:

(1) We drive DOPs for the TOA and TDOA based methods depending on different NLOS cases. The performance of the TOA and TDOA based methods is investigated in terms of DOPs. These cases are general in practice environments, and the case discussed in [18] can be interpreted as a special case of our results.

(2) Some characteristics of the DOP in the GPS are introduced to investigate the performance of positioning accuracy. In addition, some new characteristics of the DOP and the relationship of the DOP between the TOA and TDOA based methods are derived. According to these characteristics, the system can improve positioning accuracy through proper choice of optimum BSs and location techniques.

The rest of this paper is organized as follows. Section II presents the definition and some characteristics of the DOP. The analysis of the TOA based methods is given in section III. The TDOA based methods are investigated in section IV. The relationship of the DOP between the TOA and TDOA based methods is derived in section $\mathrm{V}$. Conclusion is given in section VI.

\section{Dilution OF PRECISION}

In this section, we introduce the definition and some characteristics of DOP, which will be used in the later. The purpose of the DOP is to find a translation matrix between range domain errors and position domain errors. We consider the following model:

$$
\Delta r=H \Delta \theta
$$

where $\Delta r$ is a range error vector, $\Delta \theta$ is a position error vector and $H$ is a translation matrix. The original DOP was derived assuming that the measurement noises are uncorrelated to one another identically distributed with the noise covariance $R_{r}=I \sigma_{r}{ }^{2}$. The DOP is defined as [14]:

$$
D O P=\sqrt{\operatorname{trace}\left(D_{1}\right)}=\sqrt{\operatorname{trace}\left(\left(H^{T} H\right)^{-1}\right)}
$$

where $D_{1}$ is the DOP matrix.

However, when the position of MS is estimated in wireless communication systems, there are significant variations in the error distributions due to NLOS propagation and the DOP is no longer a proper measure of the user position domain variance. To address this problem, the KDOP and weighted DOP (WDOP) were proposed in [15]. The KDOP is a variant of the DOP with the least square (LS) solution and the WDOP is with the weighted least square (WLS) solution. From (1), the LS solution for $\Delta \theta$ is given by:

$$
\Delta \theta=\left(H^{T} H\right)^{-1} H^{T} \Delta r
$$

Its corresponding covariance matrix can be written as:

$$
\operatorname{cov}(\Delta \theta)=\left(H^{T} H\right)^{-1} H^{T} R_{r} H\left(H^{T} H\right)^{-1}
$$

where $R_{r}$ is the covariance matrix of range errors. The KDOP is defined as [15]:

$$
K D O P=\sqrt{\operatorname{trace}(\operatorname{cov}(\Delta \theta))}=\sqrt{\operatorname{trace}\left(D_{2}\right)}
$$

$$
=\sqrt{\operatorname{trace}\left(\left(H^{T} H\right)^{-1} H^{T} R_{r} H\left(H^{T} H\right)^{-1}\right)}
$$

where $D_{2}$ is the KDOP matrix. The WLS solution for $\Delta \theta$ of (1) is given by:

$$
\Delta \theta=\left(H^{T} R_{r}^{-1} H\right)^{-1} H^{T} R_{r}^{-1} \Delta r
$$

The covariance matrix of $\Delta \theta$ can be written as:

$$
\begin{aligned}
\operatorname{cov}(\Delta \theta) & =\left(H^{T} R_{r}^{-1} H\right)^{-1} H^{T} R_{r}^{-1} R_{r} R_{r}^{-1} H\left(H^{T} R_{r}^{-1} H\right)^{-1} \\
& =\left(H^{T} R_{r}^{-1} H\right)^{-1} H^{T} R_{r}^{-1} H\left(H^{T} R_{r}^{-1} H\right)^{-1} \\
& =\left(H^{T} R_{r}^{-1} H\right)^{-1}
\end{aligned}
$$

The WDOP is defined as [15]:

$$
\begin{aligned}
W D O P & =\sqrt{\operatorname{trace}(\operatorname{cov}(\Delta \theta))}=\sqrt{\operatorname{trace}\left(D_{3}\right)} \\
& =\sqrt{\operatorname{trace}\left(\left(H^{T} R_{r}^{-1} H\right)^{-1}\right)}
\end{aligned}
$$

where $D_{3}$ is the WDOP matrix. It can be seen from (5) and (8) that the KDOP and WDOP are mean square errors (MSE) of location estimator with LS solution and WLS solution, respectively. This means that the performance of location estimator can be investigated in terms of the KDOP and WDOP. Now, we present some characteristics for DOP in the following proposition.

\section{Proposition 1}

If covariance matrix $R_{r}$ is diagonal, WDOP is monotonically decreasing in respect of the number of BSs.

Proof: See [15].

Proposition 1 shows that the performance of the TOA with a diagonal covariance matrix will become better as the number of BSs increases and the WLS method is used.

Proposition 2

If range errors are independent identically distributed (i.i.d) random variables and covariance matrix $R_{r}$ is diagonal, then the KDOP equals WDOP.

Proof: If range errors are i.i.d random variables and covariance matrix $R_{r}$ is diagonal, then range errors will have a covariance matrix $R_{r}=I \sigma_{r}{ }^{2}$. Substituting $R_{r}=I \sigma_{r}{ }^{2}$ into (5) and (8), the WDOP and KDOP can be rewritten as:

$$
\begin{gathered}
\text { WDOP }=\sqrt{\operatorname{trace}\left[\left(H^{T} I\left(1 / \sigma_{r}{ }^{2}\right) H\right)^{-1}\right]} \\
=\sqrt{\operatorname{trace}\left[\left(\left(H^{T} H\right)^{-1} I \sigma_{r}^{2}\right)\right]} \\
\text { KDOP }=\sqrt{\operatorname{trace}\left(\left(H^{T} H\right)^{-1} H^{T} I \sigma_{r}{ }^{2} H\left(H^{T} H\right)^{-1}\right)} \\
=\sqrt{\operatorname{trace}\left(\left(H^{T} H\right)^{-1} H^{T} H\left(H^{T} H\right)^{-1} I \sigma_{r}{ }^{2}\right)} \\
=\sqrt{\operatorname{trace}\left(\left(H^{T} H\right)^{-1} I \sigma_{r}{ }^{2}\right)} \\
\text { Hence, } \quad W D O P=K D O P
\end{gathered}
$$

Proposition 2 means that the performance of the LS method and WLS method are equal under the conditions of Proposition 2.

\section{Proposition 3}

For i.i.d errors variables and diagonal covariance matrix, the lowest attainable WDOP and KDOP in N-sided regular 
polygon, $N>3$, locate at the centre of the polygon, and its corresponding KDOP and WDOP matrix are $\left[D_{2}\right]_{2 \times 2}=\left[D_{3}\right]_{2 \times 2}=\operatorname{diag}\left(\left[\frac{2}{N} \frac{2}{N}\right]\right) I \sigma_{r}{ }^{2}$.

Proof: For i.i.d errors variables, it can be seen from [16] that $\left[\left(H^{T} H\right)^{-1}\right]_{2 \times 2}=\operatorname{diag}\left(\left[\frac{2}{N} \frac{2}{N}\right]\right)$ when MS locates at the centre of the $\mathrm{N}$-sided regular polygon.

From (11), we have:

$$
\begin{aligned}
{\left[D_{2}\right]_{2 \times 2} } & =\left[D_{3}\right]_{2 \times 2}=\left[\left(H^{T} H\right)^{-1}\right]_{2 \times 2} I \sigma_{r}{ }^{2} \\
& =\operatorname{diag}\left(\left[\frac{2}{N} \frac{2}{N}\right]\right) I \sigma_{r}{ }^{2}
\end{aligned}
$$

It can be included from Proposition 3 that the lowest attainable WDOP and KDOP in N-sided regular polygon locate at the centre of the polygon. It should be noted that the DOP, KDOP and WDOP are all based on the assumption that range errors have zero mean. In practice, NLOS errors are positive random variables. We will deal with this case in the later section depending on different NLOS environments.

\section{ANALYSIS OF THE TOA BASED METHOD}

In this section we present the analysis results of the TOA based methods. The analysis results are derived for two main different cases depending on whether the number of LOS BSs is less than three or not. The first main case includes two small cases depending on whether NLOS errors are i.i.d random variables or not.

Assuming that $(x, y)$ is the position of the MS, and $\left(x_{i}, y_{i}\right)$ is the position of the $i$ th $\mathrm{BS}$. The $i$ th range measurement $\widehat{r}_{i}$ is modeled as:

$$
\widehat{r}_{i}=r_{i}+n_{i}+e_{i}=\sqrt{\left(x-x_{i}\right)^{2}+\left(y-y_{i}\right)^{2}}+n_{i}+e_{i}
$$

where $r_{i}$ is the true distance between the MS and BS $i, N$ is the number of BSs, $n_{i}$ represents the standard measurement noise subjected to zero mean Gaussian distribution with variance $\delta_{i}^{2}, e_{i}$ is a positive random variable representing the error due to NLOS propagation, which subject to different statistical distributions with mean $\bar{e}_{i}$ and variance $\sigma_{i}^{2}$ in the different channel environments [24], such as Exponential, Uniform, and Delta distribution.

3.1 The case where the number of LOS BSs is less than three

When the number of LOS BSs is less than three, NLOS errors $e_{i}$ cannot be estimated. Since the number of unknown parameters, including the position of the MS and NLOS errors $e_{i}$, is more than the number of measurement equation. Thus, the CRLB derived in [18] cannot be available. Here, we derive DOPs depending on whether NLOS errors are i.i.d random variables or not.

3.1.1 NLOS errors are not i.i.d random variables

When NLOS errors are not i.i.d random variables and there are not prior statistics of NLOS errors, the location estimator will be biased. The linearized measurement equation for (13) is written as:

$$
\Delta r_{i}=\frac{\partial r_{i}}{\partial x} \Delta x+\frac{\partial r_{i}}{\partial y} \Delta y
$$

where $\Delta r_{i}$ is range error, $\Delta x$ and $\Delta y$ are the estimation errors of $x$ and $y$, respectively. Eqn (14) can be expressed in matrix form:

$$
\Delta r=H_{1} \Delta \theta_{1}
$$

where $\Delta r=\left[\begin{array}{c}\Delta r_{1} \\ \vdots \\ \Delta r_{N}\end{array}\right] \quad, \quad H_{1}=\left[\begin{array}{cc}\frac{\partial r_{1}}{\partial x} & \frac{\partial r_{1}}{\partial y} \\ \vdots & \vdots \\ \frac{\partial r_{N}}{\partial x} & \frac{\partial r_{N}}{\partial y}\end{array}\right] \quad, \quad$ and $\Delta \theta_{1}=\left[\begin{array}{c}\Delta x \\ \Delta y\end{array}\right]$. The LS solution for $\Delta \theta_{1}$ is given by:

$$
\Delta \theta_{1}=\left(H_{1}^{T} H_{1}\right)^{-1} H_{1}^{T} \Delta r
$$

The mean matrix and $E\left(\Delta \theta_{1} \Delta \theta_{1}^{T}\right)$ of $\Delta \theta_{1}$ can be written as:

$$
\begin{gathered}
E\left(\Delta \theta_{1}\right)=\left(H_{1}^{T} H_{1}\right)^{-1} H_{1}{ }^{T} E(\Delta r) \\
=\left(H_{1}^{T} H_{1}\right)^{-1} H_{1}^{T}\left[\begin{array}{lll}
\bar{e}_{1} & \cdots & \bar{e}_{N}
\end{array}\right]^{T} \\
E\left(\Delta \theta_{1} \Delta \theta_{1}^{T}\right)=\left(H_{1}{ }^{T} H_{1}\right)^{-1} H_{1}{ }^{T} R_{r} H_{1}\left(H_{1}{ }^{T} H_{1}\right)^{-1}
\end{gathered}
$$

where $E\left(\Delta r_{i} \Delta r_{j}\right)=\left\{\begin{array}{c}\delta_{i}^{2}+\sigma_{i}^{2}+\bar{e}_{i}^{2}, j=i \\ \bar{e}_{i} \bar{e}_{j}, j \neq i\end{array}\right.$,

$$
R_{r}=\left[\begin{array}{ccc}
E\left(\Delta r_{1}^{2}\right) & \cdots & E\left(\Delta r_{1} \Delta r_{N}\right) \\
\vdots & \vdots & \vdots \\
E\left(\Delta r_{N} \Delta r_{1}\right) & \cdots & E\left(\Delta r_{N}{ }^{2}\right)
\end{array}\right]
$$

The KDOP can be obtained:

$$
\begin{aligned}
K D O P & =\sqrt{\operatorname{trace}\left[\operatorname{cov}\left(\Delta \theta_{1}\right)\right]} \\
& =\sqrt{\operatorname{trace}\left[E\left(\Delta \theta_{1} \Delta \theta_{1}^{T}\right)-E\left(\Delta \theta_{1}\right) E\left(\Delta \theta_{1}\right)^{T}\right]}
\end{aligned}
$$

It can be seen from (17) that the location estimator is biased and the mean and variance of location errors can be computed by (17) and (19), respectively.

In some cases, we may have the mean and variance of $e_{i}$ based on field experiments or some statistical scattering model. Then an unbiased estimator and a higher positioning accuracy should be expected. There, we discuss how such prior information should be incorporated in the analysis. Suppose that the mean $\bar{e}_{i}$ and variance $\sigma_{i}^{2}$ of $e_{i}$ can be obtained beforehand. From (13), we have:

$$
\widehat{r}_{i}^{\prime}=\widehat{r}_{i}-\bar{e}_{i}=r_{i}+n_{i}+e_{i}^{\prime}, i=1, \cdots, N
$$


where $e_{i}^{\prime}=e_{i}-\bar{e}_{i}$. With the mean $\bar{e}_{i}$ and variance $\sigma_{i}^{2}$ of $e_{i}$ and from (8), the WDOP can be computed as:

$$
\begin{aligned}
W D O P & =\sqrt{\operatorname{trace}\left(\operatorname{cov}\left(\Delta \theta_{1}\right)\right)} \\
& =\sqrt{\operatorname{trace}\left(\left(H_{1}^{T} R_{r}{ }^{-1} H_{1}\right)^{-1}\right)}
\end{aligned}
$$

where $R_{r^{\prime}}=\operatorname{diag}\left(\left[\begin{array}{lll}\delta_{1}^{2}+\sigma_{1}^{2} & \cdots & \delta_{N}{ }^{2}+\sigma_{N}{ }^{2}\end{array}\right]\right)$.

\subsubsection{NLOS errors are i.i.d random variables}

In most cases, the range measurement noise may have an identical variance $\delta^{2}$, and NLOS errors may be i.i.d random variables with the mean $\bar{e}$ and the variance $\sigma^{2}$. In these cases, an unbiased location estimator of MS position can be obtained without the prior knowledge of NLOS errors. There, (13) can be rewritten as:

$$
\widehat{r}_{i}=r_{i}+n_{i}+e_{i}=r_{i}+n_{i}+\bar{e}+e_{i}^{\prime}, i=1, \cdots, N, N>3
$$

where $e_{i}^{\prime}=e_{i}-\bar{e}_{i}$. Let $\bar{e}$ be an unknown parameter. If the number of all BSs is more than three, then $\bar{e}$ can be estimated. Since the number of measurement equations is more than the number of unknown parameters. From (22), the $H$ matrix and $\Delta \theta$ matrix in (1) can be rewritten as:

$$
H_{2}=\left[\begin{array}{ccc}
\frac{\partial r_{1}}{\partial x} & \frac{\partial r_{1}}{\partial y} & 1 \\
\vdots & \vdots & \vdots \\
\frac{\partial r_{N}}{\partial x} & \frac{\partial r_{N}}{\partial x} & 1
\end{array}\right], \Delta \theta_{2}=\left[\begin{array}{c}
\Delta x \\
\Delta y \\
\Delta \bar{e}
\end{array}\right]
$$

The LS solution for $\Delta \theta_{2}$ is given by:

$$
\Delta \theta_{2}=\left(H_{2}{ }^{T} H_{2}\right)^{-1} H_{2}{ }^{T} \Delta r
$$

The mean matrix of $\Delta \theta_{2}$ can be written as:

$$
\begin{aligned}
& E\left(\Delta \theta_{2}\right)=\left(H_{2}{ }^{T} H_{2}\right)^{-1} H_{2}{ }^{T} E(\Delta r)
\end{aligned}
$$

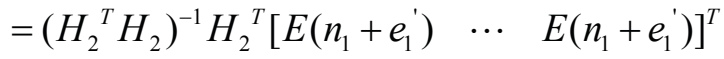

$$
\begin{aligned}
& =0
\end{aligned}
$$

Equation (25) shows that the estimator will be an unbiased estimator, while let $\bar{e}$ be an unknown parameter and estimating it. From (11), the KDOP and WDOP can be computed as:

$$
\begin{aligned}
W D O P & =K D O P=\sqrt{\operatorname{trace}\left[\operatorname{cov}\left(\Delta \theta_{2}\right)\right]} \\
& =\sqrt{\operatorname{trace}\left[\left(H_{2}{ }^{T} R_{r}{ }^{-1} H_{2}\right)^{-1}\right]}
\end{aligned}
$$

where

$$
R_{r}=\left[\begin{array}{ccc}
E\left[\left(n_{1}+e_{1}^{\prime}\right)^{2}\right] & \cdots & E\left[\left(n_{1}+e_{1}^{\prime}\right)\left(n_{N}+e_{N}^{\prime}\right)\right] \\
\vdots & \vdots & \vdots \\
E\left[\left(n_{N}+e_{N}^{\prime}\right)\left(n_{1}+e_{1}^{\prime}\right)\right] & \cdots & E\left[\left(n_{N}+e_{N}^{\prime}\right)^{2}\right]
\end{array}\right]
$$

$$
=\left[\begin{array}{ccc}
\delta^{2}+\sigma^{2} & \cdots & 0 \\
\vdots & \vdots & \vdots \\
0 & \cdots & \delta^{2}+\sigma^{2}
\end{array}\right]
$$

3.2 The case where the number of LOS BSs is three or more

The authors in [18] drive the CRLB on geolocation accuracy in mixed NLOS/LOS environments, under the assumption that there are three or more LOS BSs and NLOS errors can be estimated by LOS BSs. In this section, we also consider this case and analyze positioning accuracy based on the DOP. The relationship between the DOP and CRLB drived in [18] is also presented in the following analysis.

Here, denote the set of $\mathrm{M}$ BSs by $N L$, which can be labeled as $\{1,2, \cdots, M\}$ without loss generality. The signals received by the BSs in this set are NLOS signals. The complement of $N L$, denoted by $L$, which can be labeled as $\{M+1, M+2, \cdots, N\}$, is the set of LOS stations. The parameters to be estimated are the MS position and NLOS propagation induced path length errors, $\theta_{3}=\left[x, y, e_{1}, e_{2}, \cdots, e_{M}\right]^{T}$. The range measurement can be rewritten as:

$$
\widehat{r}_{i}=\left\{\begin{array}{c}
r_{i}+n_{i}+e_{i}, i \in N L \\
r_{i}+n_{i}, i \in L
\end{array}\right.
$$

The $H$ matrix and $\Delta \theta$ matrix in (1) can be rewritten as:

$$
\begin{gathered}
\Delta \theta_{3}=\left[\begin{array}{lllll}
\Delta x & \Delta y & \Delta e_{1} & \cdots & \Delta e_{M}
\end{array}\right]^{T}, \\
H_{3}=\left[\begin{array}{ccccc}
\frac{\partial r_{1}}{\partial x} & \frac{\partial r_{1}}{\partial y} & 1 & \cdots & 0 \\
\vdots & \vdots & \vdots & \ddots & \vdots \\
\frac{\partial r_{M}}{\partial x} & \frac{\partial r_{M}}{\partial y} & 0 & \cdots & 1 \\
\frac{\partial r_{M+1}}{\partial x} & \frac{\partial r_{M+1}}{\partial y} & 0 & \cdots & 0 \\
\vdots & \vdots & \vdots & \ddots & \vdots \\
\frac{\partial r_{N}}{\partial x} & \frac{\partial r_{N}}{\partial y} & 0 & \cdots & 0
\end{array}\right]=\left[\begin{array}{cc}
H_{N L} & I_{M} \\
H_{L} & 0
\end{array}\right]
\end{gathered}
$$

From (5), the KDOP can be computed by the LS solution:

$K D O P=\sqrt{\operatorname{trace}\left[\left(H_{3}{ }^{T} H_{3}\right)^{-1} H_{3}{ }^{T} R_{r} H_{3}\left(H_{3}{ }^{T} H_{3}\right)^{-1}\right]}$ (30) where

$$
\begin{gathered}
R_{r}=\left[\begin{array}{cc}
\Lambda_{N L} & 0 \\
0 & \Lambda_{L}
\end{array}\right], \Lambda_{N L}=\operatorname{diag}\left(\left[\begin{array}{lll}
\delta_{1}^{2} & \cdots & \delta_{M}{ }^{2}
\end{array}\right]\right) \\
\Lambda_{L}=\operatorname{diag}\left(\left[\begin{array}{llll}
\delta_{M+1}{ }^{2} & \cdots & \left.\left.\delta_{N}{ }^{2}\right]\right)
\end{array}\right.\right.
\end{gathered}
$$

From (8), the WDOP can be computed by the WLS solution:

$$
W D O P=\sqrt{\operatorname{trace}\left[\left(\mathrm{H}_{3}{ }^{T} \mathrm{R}_{r}^{-1} \mathrm{H}_{3}\right)^{-1}\right]}
$$

The relationship between WDOP and CRLB is presented in the proposition 4.

Proposition 4

When there are three or more LOS BSs, the WDOP equals the CRLB derived in [18]. 
Proof: the CRLB matrix is defined as the inverse of the Fisher information matrix (FIM) $J_{\theta}$ :

$$
E\left(\Delta \theta_{3} \Delta \theta_{3}^{T}\right) \geq J_{\theta}^{-1}
$$

where " $A \geq B$ " should be interpreted as matrix $(A-B)$ is non-negative definite. The FIM is determined by:

$$
J_{\theta}=E\left[\frac{\partial}{\partial \theta} \ln f(\widehat{r} \mid \theta)\left(\frac{\partial}{\partial \theta} \ln f(\widehat{r} \mid \theta)\right)^{T}\right]
$$

where $f(\hat{r} \mid \theta)$ is the joint probability density function of $\left[\begin{array}{lll}\widehat{r}_{1} & \cdots & \widehat{r}_{N}\end{array}\right]$ conditioned on $\theta$. With (28), we have:

$$
f(\widehat{r} \mid \theta) \propto \exp \left\{(\hat{r}-r(\theta))^{T} R_{r}^{-1}(\widehat{r}-r(\theta))\right\}
$$

With the chain rule, we have:

$$
\frac{\partial \ln f(\widehat{r} \mid \theta)}{\partial \theta}=H_{3}^{T} R_{r}^{-1} \Delta r
$$

Substituting (36) into (34), we have:

$$
\begin{aligned}
J_{\theta} & =E\left[\frac{\partial}{\partial \theta} \ln f(\widehat{r} \mid \theta)\left(\frac{\partial}{\partial \theta} \ln f(\hat{r} \mid \theta)\right)^{T}\right] \\
& =H_{3}{ }^{T} R_{r}^{-1} E\left(\Delta r \Delta r^{T}\right) R_{r}^{-1} H_{3} \\
& =H_{3}{ }^{T} R_{r}{ }^{-1} R_{r} R_{r}{ }^{-1} H_{3} \\
& =H_{3}{ }^{T} R_{r}{ }^{-1} H_{3}
\end{aligned}
$$

Hence, the WDOP equals the CRLB.

In other words, the WLS solution can attain the CRLB for position estimates in an NLOS environment when the number of LOS BSs is three or more. It can be concluded from Proposition 4 that related discussions given in [18] can be interpreted as special cases of our results.

The characteristic of WDOP matrix is discussed below:

Proposition 5

When there are three or more LOS BSs, the WDOP matrix for the MS position is

$$
\left[\left(H_{3}{ }^{T} R_{r}{ }^{-1} H_{3}\right)^{-1}\right]_{2 \times 2}=\left(H_{L}{ }^{T} \Lambda_{L}{ }^{-1} H_{L}\right)^{-1}
$$

Proof: Substituting (29) into $\left(\mathrm{H}_{3}{ }^{T} \mathrm{R}_{r}{ }^{-1} \mathrm{H}_{3}\right)^{-1}$, we have:

$$
\begin{gathered}
\left(H_{3}{ }^{T} R_{r}{ }^{-1} H_{3}\right)^{-1}=\left[\begin{array}{cc}
A_{11} & A_{12} \\
A_{21} & A_{22}
\end{array}\right]^{-1} \\
=\left[\begin{array}{cc}
H_{N L}{ }^{T} \Lambda_{N L}{ }^{-1} H_{N L}+H_{L}{ }^{T} \Lambda_{L}{ }^{-1} H_{L} & H_{N L}{ }^{T} \Lambda_{N L}{ }^{-1} \\
\Lambda_{N L}{ }^{-1} H_{N L} & \Lambda_{N L}{ }^{-1}
\end{array}\right]^{-1}
\end{gathered}
$$

Using the matrix inversion lemma [26], we have:

$$
\begin{aligned}
& {\left[\begin{array}{cc}
A_{11} & A_{12} \\
A_{21} & A_{22}
\end{array}\right]^{-1} } \\
= & {\left[\begin{array}{cc}
\left(A_{11}-A_{12} A_{22}{ }^{-1} A_{21}\right)^{-1} & A_{11}{ }^{-1} A_{12}\left(A_{21} A_{11}{ }^{-1} A_{12}-A_{22}\right)^{-1} \\
\left(A_{21} A_{11}{ }^{-1} A_{12}-A_{22}\right)^{-1} A_{21} A_{11}{ }^{-1} & \left(A_{22}-A_{21} A_{11}{ }^{-1} A_{12}\right)^{-1}
\end{array}\right] } \\
\left(A_{11}-A_{12} A_{22}{ }^{-1} A_{21}\right)^{-1} & \left(H_{N L}{ }^{T} \Lambda_{N L}{ }^{-1} H_{N L}+H_{L}{ }^{T} \Lambda_{L}{ }^{-1} H_{L}-H_{N L}{ }^{T} \Lambda_{N L}{ }^{-1} \Lambda_{N L} \Lambda_{N L}{ }^{-1} H_{N L}\right)^{-1} \\
= & \left(H_{N L}{ }^{T} \Lambda_{N L}{ }^{-1} H_{N L}+H_{L}{ }^{T} \Lambda_{L}{ }^{-1} H_{L}-H_{N L}{ }^{T} \Lambda_{N L}{ }^{-1} H_{N L}\right)^{-1}
\end{aligned}
$$

$$
=\left(H_{L}{ }^{T} \Lambda_{L}{ }^{-1} H_{L}\right)^{-1}
$$

Hence,

$$
\begin{aligned}
{\left[\left(H_{3}{ }^{T} R_{r}{ }^{-1} H_{3}\right)^{-1}\right]_{2 \times 2} } & =\left(A_{11}-A_{12} A_{22}{ }^{-1} A_{21}\right)^{-1} \\
& =\left(H_{L}{ }^{T} \Lambda_{L}{ }^{-1} H_{L}\right)^{-1}
\end{aligned}
$$

Another proof is provided in [18].

It can be concluded from Proposition 5 that the positioning accuracy depends only on the LOS signals when there are sufficient LOS BSs (three or more).

\section{ANALYSIS OF THE TDOA BASED METHOD}

Here, we present the main results of the analysis of the TDOA based method in NLOS environments.

The different range measurements $\widehat{r}_{i N}$ can be model as:

$$
\hat{r}_{i N}=\widehat{r}_{i}-\widehat{r}_{N}=r_{i}-r_{N}+\left(n_{i}-n_{N}\right)+\left(e_{i}-e_{N}\right)
$$

4.1 The case where the number of LOS BSs is less than three 4.1.1 NLOS errors ar not i.i.d random variables.

From (42), the $H$ matrix and $\Delta \theta$ matrix in (1) can be rewritten as:

$$
H_{4}=\left[\begin{array}{cc}
\frac{\partial r_{1}}{\partial x}-\frac{\partial r_{N}}{\partial x} & \frac{\partial r_{1}}{\partial y}-\frac{\partial r_{N}}{\partial y} \\
\vdots & \vdots \\
\frac{\partial r_{N-1}}{\partial x}-\frac{\partial r_{N}}{\partial x} & \frac{\partial r_{N-1}}{\partial y}-\frac{\partial r_{N}}{\partial y}
\end{array}\right], \Delta \theta_{4}=\left[\begin{array}{c}
\Delta x \\
\Delta y
\end{array}\right]
$$

$\Delta \theta_{4}$ 's corresponding mean matrix and $E\left(\Delta \theta_{4} \Delta \theta_{4}^{T}\right)$ can be written as:

$$
\begin{aligned}
& E\left(\Delta \theta_{4}\right)=\left(H_{4}{ }^{T} H_{4}\right)^{-1} H_{4}{ }^{T} E(\Delta r)
\end{aligned}
$$

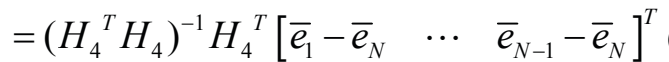

$$
\begin{aligned}
& E\left(\Delta \theta_{4} \Delta \theta_{4}^{T}\right)=\left(H_{4}^{T} H_{4}\right)^{-1} H_{4}{ }^{T} R_{d r} H_{4}\left(H_{4}{ }^{T} H_{4}\right)^{-1} \\
& \text { where } R_{d r}=\left[\begin{array}{ccc}
E\left(\Delta{r_{1 N}}^{2}\right) & \cdots & E\left(\Delta r_{1 N} \Delta r_{(N-1) N}\right) \\
\vdots & \vdots & \vdots \\
E\left(\Delta r_{(N-1) N} \Delta r_{1 N}\right) & \cdots & E\left(\Delta r_{(N-1) N}{ }^{2}\right)
\end{array}\right] \text {. } \\
& E\left(\Delta r_{i N}{ }^{2}\right)=E\left(\left(n_{i}-n_{N}+e_{i}-e_{N}\right)^{2}\right) \\
& =E\left(n_{i}^{2}-2 n_{i} n_{N}+n_{N}^{2}+2\left(n_{i} e_{i}-n_{i} e_{N}-n_{N} e_{i}+n_{N} e_{N}\right)\right. \\
& \left.+e_{i}^{2}-2 e_{i} e_{N}+e_{N}^{2}\right) \\
& =\delta_{i}^{2}+\delta_{N}^{2}+E\left(e_{i}^{2}\right)-2 \bar{e}_{i} \bar{e}_{N}+E\left(e_{N}^{2}\right) \\
& =\delta_{i}^{2}+\delta_{N}{ }^{2}+\bar{e}_{i}^{2}+\sigma_{i}{ }^{2}-2 \bar{e}_{i} \bar{e}_{N}+\bar{e}_{N}{ }^{2}+\sigma_{N}{ }^{2} \\
& E\left(\Delta r_{i N} \Delta r_{j N}\right)=E\left(\left(n_{i}-n_{N}+e_{i}-e_{N}\right)\left(n_{j}-n_{N}+e_{j}-e_{N}\right)\right) \\
& =E\left(n_{N}{ }^{2}+e_{i} e_{j}-e_{i} e_{N}-e_{N} e_{j}+e_{N}{ }^{2}\right) \\
& =\delta_{N}{ }^{2}+\bar{e}_{i} \bar{e}_{j}-\bar{e}_{i} \bar{e}_{N}-\bar{e}_{N} \bar{e}_{j}+\bar{e}_{N}{ }^{2}+\sigma_{N}{ }^{2}
\end{aligned}
$$

$$
K D O P=\sqrt{\operatorname{trace}\left[\operatorname{cov}\left(\Delta \theta_{4}\right)\right]}
$$




$$
=\sqrt{\operatorname{trace}\left[E\left(\Delta \theta_{4} \Delta \theta_{4}^{T}\right)-E\left(\Delta \theta_{4}\right) E\left(\Delta \theta_{4}\right)^{T}\right]}
$$

It can be seen from (44) that the location estimator will be biased if $\bar{e}_{i} \neq \bar{e}_{j}, i \neq j$. If we have the mean and variance of $e_{i}$ based on field experiments or some statistical scattering model, then an unbiased estimator and a higher positioning accuracy should be expected. The different range measurements model can be written as:

$\widehat{r}_{i N}^{\prime}=\widehat{r}_{i}-\bar{e}_{i}-\left(\widehat{r}_{N}-\bar{e}_{N}\right)=r_{i}-r_{N}+n_{i}-n_{N}+e_{i}^{\prime}-e_{N}^{\prime}(48)$ where $e_{i}{ }^{\prime}=e_{i}-\bar{e}_{i}$. With the mean $\bar{e}_{i}$ and variance $\sigma_{i}^{2}$ of $e_{i}$ and from (8), the WDOP can be computed as:

$$
\begin{aligned}
\text { WDOP } & =\sqrt{\operatorname{trace}\left(\operatorname{cov}\left(\Delta \theta_{4}\right)\right)} \\
& =\sqrt{\operatorname{trace}\left(\left(H_{4}{ }^{T} R_{d r}{ }^{-1} H_{4}\right)^{-1}\right)}
\end{aligned}
$$

where $R_{d r^{\prime}}=$

$$
\left[\begin{array}{ccc}
\delta_{1}{ }^{2}+\delta_{N}{ }^{2}+\sigma_{1}{ }^{2}+\sigma_{N}{ }^{2} & \cdots & \delta_{N}{ }^{2}+{\sigma_{N}}^{2} \\
\vdots & \vdots & \vdots \\
\delta_{N}{ }^{2}+{\sigma_{N}}^{2} & \cdots & \delta_{N-1}{ }^{2}+{\delta_{N}}^{2}+{\sigma_{N-1}}^{2}+{\sigma_{N}}^{2}
\end{array}\right]
$$

\section{1.2 NLOS errors are i.i.d random variables}

If NLOS errors are i.i.d random variables, then we can get an unbiased estimator without prior knowledge. The different range measurements model can be rewritten as:

$$
\begin{aligned}
\widehat{r}_{i N} & =\widehat{r}_{i}-\widehat{r}_{N}=r_{i}-r_{N}+n_{i}-n_{N}+\bar{e}_{i}-\bar{e}_{N}+e_{i}^{\prime}-e_{N}{ }^{\prime} \\
& =r_{i}-r_{N}+n_{i}-n_{N}+e_{i}^{\prime}-e_{N}^{\prime}
\end{aligned}
$$

Equation (44) becomes:

$E\left(\Delta \theta_{4}\right)=\left(H_{4}{ }^{T} H_{4}\right)^{-1} H_{4}{ }^{T} E(\Delta r)$

$=\left(H_{4}{ }^{T} H_{4}\right)^{-1} H_{4}{ }^{T} E\left[\begin{array}{lll}e_{1}^{\prime}-e_{N}^{\prime} & \cdots & e_{N-1}^{\prime}-e_{N}^{\prime}\end{array}\right]^{T}$

$=0$

The KDOP can be computed as:

$$
K D O P=\sqrt{\operatorname{trace}\left[\left(H_{4}{ }^{T} H_{4}\right)^{-1} H_{4}{ }^{T} R_{d r} H_{4}\left(H_{4}{ }^{T} H_{4}\right)^{-1}\right]}
$$

where $R_{d r}=$

$$
\left[\begin{array}{ccc}
\delta_{1}^{2}+{\delta_{N}}^{2}+\sigma_{1}^{2}+{\sigma_{N}}^{2} & \cdots & \delta_{N}{ }^{2}+\sigma_{N}{ }^{2} \\
\vdots & \vdots & \vdots \\
\delta_{N}{ }^{2}+{\sigma_{N}}^{2} & \cdots & \delta_{N-1}{ }^{2}+{\delta_{N}}^{2}+{\sigma_{N-1}}^{2}+{\sigma_{N}}^{2}
\end{array}\right]
$$

$$
=\left[\begin{array}{lll}
2 & 1 & 1 \\
1 & 2 & 1 \\
1 & 1 & 2
\end{array}\right]\left(\delta^{2}+\sigma^{2}\right) \text {. }
$$

The WDOP can be computed as:

$$
W D O P=\sqrt{\operatorname{trace}\left(\left(H_{4}{ }^{T} R_{d r}{ }^{-1} H_{4}\right)^{-1}\right)}
$$

It should be noted that the KDOP isn't equal to WDOP because covariance matrix is nondiagonal. 4.2 The case where the number of LOS BSs is three or more
Here, we present the results of the TDOA when the number of LOS BSs is three or more. Detailed derivations are omitted, since they exactly parallel that for the TOA in the previous section. The difference is that $H$, and $R_{r}$ vary depending on the method.

where

$$
\begin{aligned}
R_{r}= & {\left[\begin{array}{cccc}
\delta_{1}^{2}+\delta_{N}{ }^{2} & \cdots & \delta_{N}{ }^{2} \\
\vdots & \vdots & \vdots & \\
\delta_{N}{ }^{2} & \cdots & \delta_{N-1}{ }^{2}+\delta_{N}{ }^{2}
\end{array}\right], } \\
H_{4}= & {\left[\begin{array}{ccccc}
\frac{\partial r_{1}}{\partial x}-\frac{\partial r_{N}}{\partial x} & \frac{\partial r_{1}}{\partial y}-\frac{\partial r_{N}}{\partial y} & 1 & \cdots & 0 \\
\vdots & \vdots & \vdots & \ddots & \vdots \\
\frac{\partial r_{M}}{\partial x}-\frac{\partial r_{N}}{\partial x} & \frac{\partial r_{M}}{\partial y}-\frac{\partial r_{N}}{\partial y} & 0 & \cdots & 1 \\
\frac{\partial r_{M+1}}{\partial x}-\frac{\partial r_{N}}{\partial x} & \frac{\partial r_{M+1}}{\partial y}-\frac{\partial r_{N}}{\partial y} & 0 & \cdots & 0 \\
\vdots & \vdots & \vdots & \ddots & \vdots \\
\frac{\partial r_{N-1}}{\partial x}-\frac{\partial r_{N}}{\partial x} & \frac{\partial r_{N-1}}{\partial y}-\frac{\partial r_{N}}{\partial y} & 0 & \cdots & 0
\end{array}\right] . }
\end{aligned}
$$

V.

\section{RELATIONSHIP OF WDOP BETWEEN THE TOA AND TDOA}

In this section, we discuss the relationship of performance between TOA and TDOA under an identical NLOS environment. The cases discussed here depend on assumption that the number of LOS BSs is less than three.

5.1 The relationship of the WDOP between the TOA and TDOA with the prior knowledge

Proposition 6

With prior statistics of the mean and variance of NLOS errors, the WDOP of the TOA is less than the WDOP of the TDOA.

$$
\sqrt{\operatorname{trace}\left(H_{1}{ }^{T} R_{r^{\prime}}{ }^{-1} H_{1}\right)}<\sqrt{\operatorname{trace}\left(\left(H_{4}{ }^{T} R_{d r^{\prime}}{ }^{-1} H_{4}\right)^{-1}\right)}
$$

Proof: From (15) and (43), $H_{4}$ can be rewritten as:

$$
\begin{aligned}
H_{4}= & {\left[\begin{array}{ccc}
\frac{\partial r_{2}}{\partial x}-\frac{\partial r_{N}}{\partial x} & \frac{\partial r_{1}}{\partial y}-\frac{\partial r_{N}}{\partial y} \\
\vdots & \vdots \\
\frac{\partial r_{N}}{\partial x}-\frac{\partial r_{N}}{\partial x} & \frac{\partial r_{N-1}}{\partial y}-\frac{\partial r_{N}}{\partial y}
\end{array}\right] } \\
& =\left[\begin{array}{cccc}
1 & \cdots & 0 & -1 \\
\vdots & \vdots & \vdots & \vdots \\
0 & \cdots & 1 & -1
\end{array}\right]\left[\begin{array}{cc}
\frac{\partial r_{1}}{\partial x} & \frac{\partial r_{1}}{\partial y} \\
\vdots & \vdots \\
\frac{\partial r_{N}}{\partial x} & \frac{\partial r_{N}}{\partial y}
\end{array}\right]=S H_{1}
\end{aligned}
$$

And

$R_{d r^{\prime}}=$ 


$$
\begin{aligned}
& {\left[\begin{array}{ccc}
\delta_{1}^{2}+\delta_{N}{ }^{2}+\sigma_{1}{ }^{2}+{\sigma_{N}}^{2} & \cdots & \delta_{N}{ }^{2}+\sigma_{N}{ }^{2} \\
\vdots & \vdots & \vdots \\
\delta_{N}{ }^{2}+{\sigma_{N}}^{2} & \cdots & {\delta_{N-1}}^{2}+{\delta_{N}}^{2}+{\sigma_{N-1}}^{2}+{\sigma_{N}}^{2}
\end{array}\right]} \\
& =\left[\begin{array}{cccc}
1 & \cdots & 0 & -1 \\
\vdots & \vdots & \vdots & \vdots \\
0 & \cdots & 1 & -1
\end{array}\right]\left[\begin{array}{ccc}
\delta_{1}^{2}+\sigma_{1}^{2} & \cdots & 0 \\
\vdots & \vdots & \vdots \\
0 & \cdots & \delta_{N}{ }^{2}+\sigma_{N}{ }^{2}
\end{array}\right] \\
& {\left[\begin{array}{llll}
1 & \cdots & 0 & -1 \\
\vdots & \vdots & \vdots & \vdots \\
0 & \cdots & 1 & -1
\end{array}\right]^{T}} \\
& =S R_{r} S^{T}
\end{aligned}
$$

Substituting (54) and (55) into $\left(H_{4}{ }^{T} R_{d r}{ }^{-1} H_{4}\right)^{-1}$, we have:

$$
\begin{aligned}
& \left(H_{4}^{T} R_{d r^{\prime}}{ }^{-1} H_{4}\right)^{-1}=\left(H_{1}^{T} S^{T}\left(S R_{r} S^{T}\right)^{-1} S H_{1}\right)^{-1} \\
& =\left(\hat{H}_{1}^{T} \widehat{S}^{T}\left(\widehat{S} \widehat{S}^{T}\right)^{-1} \widehat{S} \hat{H}_{1}\right)^{-1} \\
& =\left(\hat{H}_{1}^{T}\left(I_{N \times N}-\frac{\hat{1} \hat{1}^{T}}{\hat{1}^{T} \hat{1}}\right) \hat{H}_{1}\right)^{-1} \\
& =\left(H_{1}^{T}\left(R_{r^{\prime}}{ }^{-1}-\frac{R_{r^{\prime}}{ }^{-1} 11^{T} R_{r^{\prime}}^{-1}}{1^{T} R_{r^{\prime}}{ }^{-1} 1}\right) H_{1}\right)^{-1}
\end{aligned}
$$

where $\hat{H}_{1}=R_{r^{\prime}}{ }^{-1 / 2} H_{1}, S=S R_{r^{\prime}}{ }^{1 / 2}, \hat{1}=R_{r^{\prime}}{ }^{-1 / 2} 1$ and 1 is a $N \times 1$ vector of one's. $\widehat{S}^{T}\left(\widehat{S} \widehat{S}^{T}\right)^{-1} \widehat{S}$ is the projection matrix to the range of $\widehat{S}$. As 1 is orthogonal to $S, \hat{1}$ is orthogonal to $\widehat{S}$. Therefore $\widehat{S}^{T}\left(\widehat{S} \widehat{S}^{T}\right)^{-1} \widehat{S}=\left(I_{N \times N}-\frac{\hat{1} \widehat{1}^{T}}{\hat{1}^{T} \widehat{1}}\right)$ [17].

Equation (56) can be rewritten as:

$$
\begin{aligned}
\left(H_{4}{ }^{T} R_{d r^{\prime}}{ }^{-1} H_{4}\right)^{-1} & =\left(H_{1}^{T} R_{r^{\prime}}{ }^{-1} H_{1}-\frac{H_{1}^{T} R_{r^{-}}{ }^{-1} 11^{T} R_{r^{-}}{ }^{-1} H_{1}}{1^{T} R_{r^{\prime}}{ }^{-1} 1}\right)^{-1} \\
& =\left(\hat{H}_{1}{ }^{T} \hat{H}_{1}-a^{T} a\right)^{-1}
\end{aligned}
$$

where $a=\frac{1^{T} R_{r^{r}}{ }^{-1} H_{1}}{\sqrt{1^{T} R_{r^{\prime}}{ }^{-1} 1}}$. Now let $\hat{H}_{1}{ }^{T} \hat{H}_{1}=P^{T} \Lambda P$. Where $P$ is an orthogonal matrix and $\Lambda$ is a diagonal matrix. It follows that:

$$
\begin{array}{r}
P^{T}\left(\hat{H}_{1}{ }^{T} \hat{H}_{1}-a^{T} a\right) P=P^{T} \hat{H}_{1}{ }^{T} \hat{H}_{1} P-(a p)^{T}(a p) \\
=\Lambda-b^{T} b=\operatorname{diag}\left(\left[\begin{array}{ll}
\lambda_{11} & \lambda_{22}
\end{array}\right]\right)-b^{T} b
\end{array}
$$

Consider the Sherman-Morrison formula [14]:

$$
\left(D+b^{T} b\right)^{-1}=D^{-1}-v\left(b^{*}\right)^{T}\left(b^{*}\right)
$$

with $b=\left[\begin{array}{ll}b_{1} & b_{2}\end{array}\right], \quad\left(b^{*}\right)=\left[\begin{array}{lll}b_{1} / \lambda_{11} & b_{2} / \lambda_{22}\end{array}\right]$,

$$
v=\frac{1}{1+\sum_{i=1}^{2} \frac{b_{i}^{2}}{\lambda_{i i}}} \text {. }
$$

From (58), we have:

$$
\begin{aligned}
& \left(D-b^{T} b\right)^{-1}=-\left(-D+b^{T} b\right)^{-1} \\
& =-\left((-D)^{-1}-v\left(b^{*}\right)^{T}\left(b^{*}\right)\right)=D^{-1}+v\left(b^{*}\right)^{T}\left(b^{*}\right)
\end{aligned}
$$

Hence,

$$
\begin{aligned}
& \operatorname{trace}\left(\left(H_{4}{ }^{T} R_{d r}{ }^{-1} H_{4}\right)^{-1}\right)=\operatorname{trace}\left(\left(\hat{H}_{1}{ }^{T} \hat{H}_{1}-b^{T} b\right)^{-1}\right) \\
& =\operatorname{trace}\left(\left(P\left(\hat{H}_{1}{ }^{T} \hat{H}_{1}-b^{T} b\right) P\right)^{-1}\right)=\operatorname{trace}\left(\left(D-b^{T} b\right)^{-1}\right) \\
& =\operatorname{trace}\left(D^{-1}\right)+\operatorname{trace}\left(v\left(b^{*}\right)^{T}\left(b^{*}\right)\right)
\end{aligned}
$$

where $\operatorname{trace}\left(D^{-1}\right)=\operatorname{trace}\left(P^{T} \hat{H}_{1}{ }^{T} \hat{H}_{1} P\right)$

$$
=\operatorname{trace}\left(\hat{H}_{1}{ }^{T} \hat{H}_{1}\right)=\operatorname{trace}\left(H_{1}{ }^{T} R_{r^{\prime}}{ }^{-1} H_{1}\right)
$$

And $\operatorname{trace}\left(v\left(b^{*}\right)^{T}\left(b^{*}\right)\right)=v \sum_{i=1}^{2}\left(\frac{b_{i}}{\lambda_{i i}}\right)^{2}=\frac{\sum_{i=1}^{2}\left(\frac{b_{i}}{\lambda_{i i}}\right)^{2}}{1+\sum_{i=1}^{2} \frac{b_{i}^{2}}{\lambda_{i i}}}$

As $\hat{H}_{1}{ }^{T} \hat{H}_{1}$ is Hermite matrix, $\lambda_{i i}>0$. Thus, $\operatorname{trace}\left(v\left(b^{*}\right)^{T}\left(b^{*}\right)\right)>0$.

Therefore

$$
\operatorname{trace}\left(\left(H_{4}^{T} R_{d r^{-1}} H_{4}\right)^{-1}\right)>\operatorname{trace}\left(H_{1}^{T} R_{r^{\prime}}{ }^{-1} H_{1}\right)
$$

5.2 The relationship of the WDOP between the TOA and TDOA when NLOS errors are i.i.d random variables.

Proposition 7

For i.i.d case, the WDOPs of the TOA and TDOA are equal.

$$
=\sqrt{\operatorname{trace}\left(\left(H_{4}{ }^{T}\left\{\left[\begin{array}{lll}
2 & 1 & 1 \\
1 & 2 & 1 \\
1 & 1 & 2
\end{array}\right]\left(\delta^{2}+\sigma^{2}\right)\right\}^{-1} H_{4}\right)^{-1}\right.}
$$

Proof: See [17]

When NLOS errors are i.i.d random variables, the relationship among WDOPs can be obtained from Proposition 6, Proposition 7, equation (49) and equation (53):

$$
\begin{gathered}
W D O P_{T O A, p r i o r}<W D O P_{T O A} \\
W D O P_{T O A}=W D O P_{T D O A, p r i o r}=W D O P_{T D O A}
\end{gathered}
$$

where $W D O P_{T O A, p r i o r}, W D O P_{T O A}, W D O P_{T D O A, p r i o r}$, and $W D O P_{T D O A}$ can be computed by (21), (26), (49), and (53), respectively, in i.i.d case.

$$
\begin{aligned}
& W_{\text {DOP }} \text { Trior } \\
& =\sqrt{\operatorname{trace}\left(\left(H_{1}^{T} H_{1}\right)^{-1} I\left(\delta^{2}+\sigma^{2}\right)\right)}
\end{aligned}
$$




$$
\begin{aligned}
& W D O P_{T O A}=\sqrt{\operatorname{trace}\left[\operatorname{cov}\left(\Delta \theta_{2}\right)\right]} \\
& =\sqrt{\operatorname{trace}\left[\left(\mathrm{H}_{2}{ }^{T} \mathrm{H}_{2}\right)^{-1} I\left(\delta^{2}+\sigma^{2}\right)\right]} \\
& W D O P_{T D O A, p r i o r}=W D O P_{T D O A} \\
& =\sqrt{\operatorname{trace}\left(\left(H_{4}^{T}\left\{\left[\begin{array}{lll}
2 & 1 & 1 \\
1 & 2 & 1 \\
1 & 1 & 2
\end{array}\right]\left(\delta^{2}+\sigma^{2}\right)\right\}^{-1} H_{4}\right)^{-1}\right)}
\end{aligned}
$$

Equation (64) shows the relationship of the WDOP between the TOA and TDOA for i.i.d case. The performances of TDOA regardless of the prior knowledge are same as the performance of TOA without the prior knowledge, and the performance of TOA with the prior knowledge has the best accuracy. This also means that the WDOP of the TDOA is monotonically decreasing in respect of the number of BSs in i.i.d case, although the covariance matrix of the TDOA is nondiagonal.

\section{CONCLUSIONS}

We present the analysis of the TOA and TDOA based methods in NLOS environments. The positioning accuracy is investigated in terms of DOPs according to different NLOS cases. Analysis results of our study include the following: In the TOA based methods, the WLS solution can attain the CRLB and the performance of the WLS solution depends only on LOS signals, when the number of LOS BSs is sufficient. If NLOS errors are not i.i.d random variables and the systems have not prior statistics of NLOS errors, then the location estimator will be biased regardless of location techniques (TOA or TDOA). On the contrary, the unbiased location estimator can be obtained. For i.i.d case and insufficient LOS Bss, the performances of TDOA regardless of the prior knowledge are same as the performance of TOA without the prior knowledge, and the performance of TOA with the prior knowledge has the best accuracy. This also means that the WDOP of the TDOA is monotonically decreasing in respect of the number of BSs in i.i.d case, although the covariance matrix of the TDOA is nondiagonal.

\section{ACKNOWLEDGMENT}

This work was supported by A*STAR SERC Intelligent Energy Distribution Systems (IEDS) Thematic Research Grant 072133 0042, Singapore.

\section{REFERENCES}

[1] FCC.: 'FCC Acts to Promote Competition and Public Safety in Enhanced Wireless 911 Services', Washington, DC: WT Rep, September 15, 1999,pp. 99-27

[2] Sayed, A.H., Tarighat, A., and Khajehnouri, N.: 'Network-Based Wireless Location: Challenges Faced in Developing Techniques for Accurate Wireless Location Information', IEEE Signal Processing Magazine, 2005, 22, (4), pp. 24-40
[3] Caffery, J.J., and Stuber, G.L.: 'Overview of radiolocation in CDMA cellular systems', IEEE Commun. Mag., 1998, 36, (4), pp. 38-45

[4] Wylie, M.P., and Holtzman, J.: 'The non-line-of-sight problem in mobile location estimation', Proc. IEEE Int. Conf. Universal Personal Commun., 1996, pp. 827-831

[5] Liao, J.F., and Chen, B.S.: ' Robust Mobile Location Estimator with NLOS Mitigation using Interacting Multiple Model Algorithm', IEEE Transactions on Wireless Communications, 2006, 5, (11), pp. 3002-3006

[6] Wuk K., and Lee J.G., and Jee, G.I.: 'The Interior-Point Method for an Optimal Treatment of Bias in Trilateration Location', IEEE Transactions on Vehicular Technology, 2006, 55, (4), pp. 1291-1301

[7] Ertel R.B., and Reed J.H.: 'Angle and time of arrival statistics for circular and elliptical scattering models', IEEE J. Sel. Areas Commun., 1999, 17, (11), pp. 1829-1840

[8] Jazzar, S.A, Caffery, J., and You, H.R.: 'A scattering model based approach to NLOS mitigation in TOA location systems', Proc. IEEE Vehicular Technology Conf., 2002, 2, pp. 861-865

[9] Wang, X., Wang, Z., and Dea, B.O.: 'A TOA-based location algorithm reducing the errors due to non-line-of-sight (NLOS) propagation', in Proc.IEEE Veh. Technol. Conf., 2001,1, (54), pp. 97-100

[10] Venkatraman, S., Caffery, J.J., and You, H.R.: 'A Novel ToA Location Algorithm using LoS Range Estimation for NLoS Environments', IEEE Trans. on Vehicular Technology, 2004, 53, (5), pp. 1515-1524.

[11] Cong, L., and Zhang, W.: 'Non-Line-of-Sight error mitigation in TDOA mobile location', Globecom 2001, 2001, pp.680-684.

[12] Spirito, A.M.: 'On the accuracy of cellular mobile station location estimation', IEEE Trans. Veh. Technol, 2001, 50, (3), pp.674-685.

[13] Masatt, P., and Rudnick, K.: 'Geometric formulas for dilution of precision calculation', J. Inst. Navigation, 1991, 37, pp.379-391.

[14] Yarlagadda, R., Ali, I., Al-Dhahir, N., and Hershey, J.: 'GPS GDOP metric', IEE Proc., Radar Sonar Navig., 2000, 147, (5), pp. 259-264.

[15] Sairo, H., Akopian, D., and Takala, J.: 'Weighted dilution of precision as quality measure in satellite positioning', IEE Proc. Radar Sonar Navig., 2003, 150, (6), pp.430-436.

[16] Levanon N.: 'Lowest GDOP in 2-D scenarios', IEE Proc. Radar Sonar Navig., 2000, 147, (3), pp.149-155.

[17] DONG, H.S, and SUNG, T.K.: 'Comparisons of Error Characteristics between TOA and TDOA Positioning', IEEE TRANSACTIONS ON AEROSPACE AND ELECTRONIC SYSTEMS, 2002, 38, (1), pp. 307311.

[18] Qi, Y., Kobayashi, H., and Suda, H.: 'Analysis of Wireless Geolocation in a Non-Line-of-Sight Environment', IEEE TRANSACTIONS ON WIRELESS COMMUNICATIONS,2006, 5, (3), pp.672-681.

[19] Borras, J., Hatrack, P., and Mandayam, N.B.: 'Decision theoretic frameworkfor NLOS identification', IEEE Vehicular Technology Conference, 1998, 2, pp. 1583-1587.

[20] Venkatraman, S., and Caffery, J.: 'A statistical approach to non-lineofsight BS identification', in Proc. the 25th International Symposium on Wireless Personal Multimedia Communications, 2002.

[21] Chan, Y.T, Tsui, W.Y., So, H.C, and Ching, P.C.: 'Time-of-Arrival Based Localization Under NLOS Conditions', IEEE Transactions on Vehicular Technology., 2006, 55, (1), pp.17-24.

[22] Venkatraman, S., Caffery, J.J., and You, H.R.: 'A Novel ToA Location Algorithm using LoS Range Estimation for NLoS Environments', IEEE Trans. on Vehicular Technology, 2004, 53, (5), pp. 1515-1524.

[23] Chaffee, J., and Abel, J.: 'GDOP and the Cramer-Rao Bound', in Proc.IEEE Position Location and Navigation Symposium, 1994, pp. 663-668.

[24] Asplind, H., et al.: 'A Channel Model for Positioning, Cost259TD(98)20', Ericsson Corp. Bern, Switzerland, 1998

[25] Tian X.H., Liao G.S.: 'An Effective TOA2Ba sed Location Method for Mitigating the Influence of the NLOS Propagation', ACTA ELECTRONICA SINICA, 2003, 31, (9), pp:1429-1432

[26] Rao, C.T.: 'Linear statistical inference and its applications', John Wily \& Sons, Inc., 1965. 